\title{
Damping parameters of a steel structure with a viscoelastic layer in the base node
}

\author{
Zdzisław Pawlak ${ }^{1, *}$, and Dawid Zwoliński ${ }^{1}$ \\ ${ }^{1}$ Faculty of Civil and Environmental Engineering, Poznań University of Technology, Piotrowo 5 \\ Street, 60-965 Poznań, Poland
}

\begin{abstract}
The aim of the article is to estimate the damping properties of a steel structural element equipped with a viscoelastic layer (VS) in the base node. The viscoelastic material has a good energy dissipation properties, has a relatively high strength, and its properties remain unchanged over the time. However, its damping properties may vary for different temperatures and excitation frequencies. In this work, the effect of frequency was examined, but the influence of temperature was omitted. For the selected viscoelastic material, laboratory tests were carried out to estimate its ability to dissipate energy in various load states. The viscoelastic material was then used as a washer in the joint connecting the steel element to the concrete base. The dynamic test and numerical analysis carried out for the structure with the viscoelastic layer allow estimation of some damping parameters for the considered system.
\end{abstract}

\section{Introduction}

In civil engineering, various methods are used to suppress excessive vibrations caused by dynamic loads. In building structures exposed to wind or earthquakes, passive and active damping systems are commonly used. Passive control techniques are often applied because they do not require any external energy supply for proper operation. Different kinds of mechanical devices, such as viscous dampers, viscoelastic dampers (VE), tuned mass dampers or base isolation systems, are applied in buildings or structures as a passive damping system. In the case of viscoelastic damper, usually a special devices containing a viscoelastic material are introduced into the bracing system of the building [1,2]. Among the others, there is a wide family of so-called base isolation systems, where the flexible isolation devices are placed horizontally at the base of the structure to decouple it from the ground [3] and to increase the natural period of vibration.

The elastomer layers are widely used as the isolation system of the base of machines producing noise or vibration. In a similar way, they are used to protect the structural elements of the building as elastomer bearings. In reinforced concrete structures (RC), elastomers are used in supports to prevent transmission of vibrations or to allow the element to deflect. When the viscoelastic material is used in a steel construction, the node is formed in such a way that the energy dissipation is obtained by the shear deformation of

*Corresponding author: zdzislaw.pawlak@put.poznan.pl 
the elastomer [4]. In this article, the viscoelastic material is used as a layer inserted under the steel plate of a structural node. First, the polymer sample was tested in a testing machine, followed by a dynamic test for a steel structure with a polymer layer in the node.

\section{Laboratory test}

The basic properties of the polymer material were determined using the ElectroPuls ${ }^{\mathrm{TM}}$ E10000 dynamic test instrument (Instron). The dynamic analysis of the structure equipped with a viscoelastic layer was carried out using the Sirius data acquisition system.

\subsection{Polymer properties}

For the laboratory tests, polyurethane with the trade name B-85 was chosen as a viscoelastic material. Some mechanical properties of the material guaranteed by the manufacturer are shown in Table 1.

Table 1. Mechanical properties of B-85 polyurethane.

\begin{tabular}{|c|c|}
\hline Characteristic & Value \\
\hline Hardness [ShA] & 89 \\
\hline Tensile strength [MPa] & 31 \\
\hline Compression set $\left(70^{\circ} \mathrm{C} / 24 \mathrm{~h}\right)[\%]$ & 15 \\
\hline Compression set $\left(20^{\circ} \mathrm{C} / 72 \mathrm{~h}\right)[\%]$ & 14 \\
\hline Rebound $[\%]$ & 43 \\
\hline
\end{tabular}

The above-mentioned properties specified by the manufacturer do not determine the ability of the polymer to dissipate energy. For this reason, the sample material was subjected to an additional laboratory test.

\subsection{Hysteresis loops}

A dynamic test of the initially compressed sample was carried out. A round plate with a diameter of $60 \mathrm{~mm}$ and a thickness of $12 \mathrm{~mm}$ was compressed with a force of different values: $0.5,1.0,2.0$ and $4.0 \mathrm{kN}$. Then the compression changes in time according to the sine function, the force with the amplitude of $500 \mathrm{~N}$ operates with different frequencies: 1.0, 5.0, 10.0 and $20.0 \mathrm{~Hz}$. Thus, for the controlled input force, the magnitude of the displacement and the phase angle were measured as a response. The ElectroPuls ${ }^{\mathrm{TM}} \mathrm{E} 10000$ test instrument must be tuned to the properties of the sample, during this process the stiffness factor is measured, in this case it was $k_{z}=13.187 \mathrm{kN} / \mathrm{mm}$. Taking into account the dimensions of the sample, it was possible to determine the Young's modulus of the tested material, which was $E=56.0 \mathrm{MPa}$. The results of the experiments are presented in the appropriate graphs in Figures 1 and 2. Figure 1 shows exemplary hysteresis loops for a compression force of $1.0 \mathrm{kN}$ and for several selected frequencies.

The diagrams presented in Fig. 1 show only the change in the compression force value in the range of its amplitude. When the initial value was $-1.0 \mathrm{kN}$ and the amplitude was equal to $500 \mathrm{~N}$, the compression varied from -0.5 to $-1.5 \mathrm{kN}$. It can be observed that as the 
frequency increases, the displacement amplitude decreases slightly, but the graphs are very similar for the tested range of 1 to $20 \mathrm{~Hz}$.

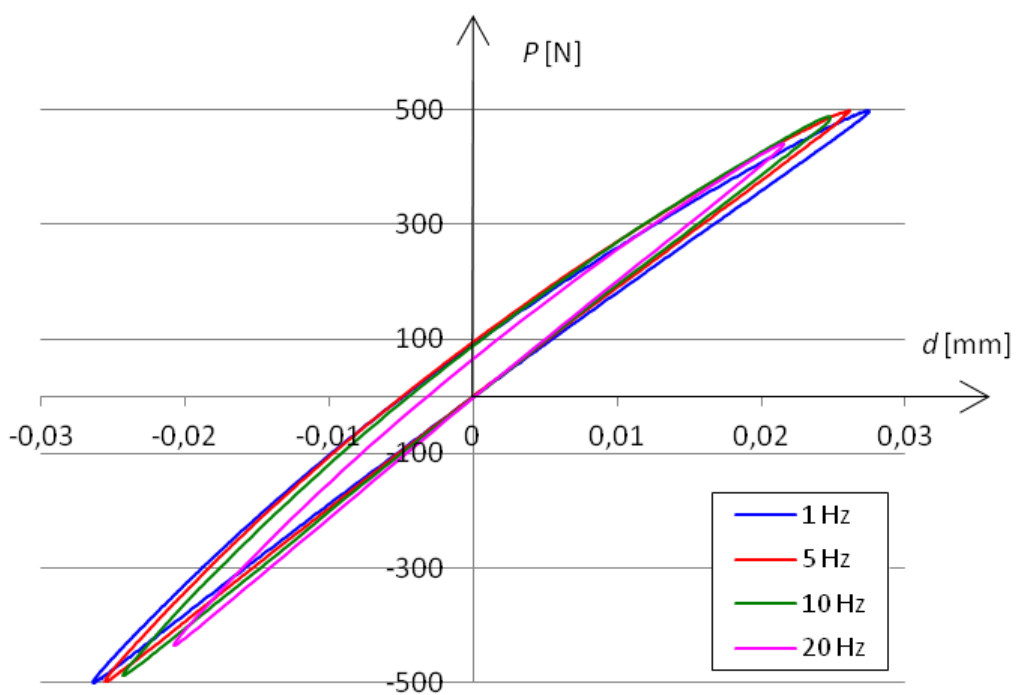

Fig. 1. Hysteresis loops for a polymer compressed by $1.0 \mathrm{kN}$ force.

The next graph (Fig. 2) presents the hysteresis loops for different compression forces. To compare the loops for different forces, the diagram only shows the displacement increment in relation to the force increment.

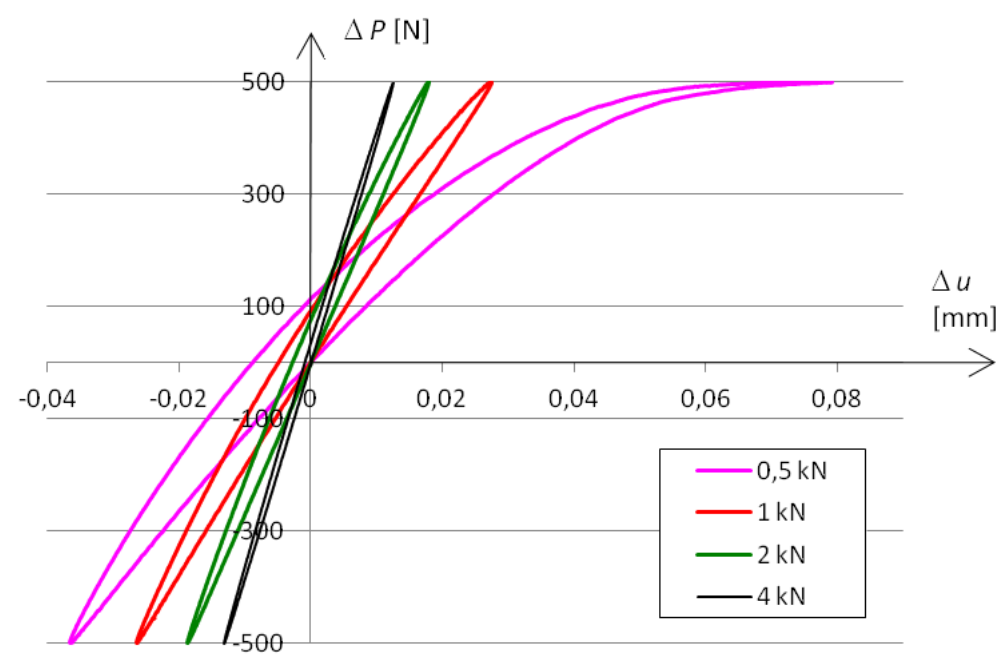

Fig. 2. Hysteresis loops for $1.0 \mathrm{~Hz}$ excitation frequency and various compression forces.

On the basis of data from Fig. 1 and 2, some damping parameters were calculated. First, the dissipation factor $\gamma$ was determined, i.e. the ratio of dissipated energy (area within the loop) to elastic energy (area under the linear force-displacement function). To calculate the damping parameter, the hysteresis loops must be formed for the actual, measured compression force, i.e. starting from $0.5,1.0,2.0$ and $4.0 \mathrm{kN}$, respectively. The results for selected frequencies and forces are presented in Table 2. 
Table 2. Damping parameters for selected compressions and excitation frequencies.

\begin{tabular}{|c|c|c|c|c|c|c|c|}
\hline Force $N[\mathbf{k N}]$ & 0.5 & \multicolumn{4}{|c|}{1.0} & 2.0 & 4.0 \\
\hline $\begin{array}{c}\text { Compression } \\
\sigma[\mathrm{MPa}]\end{array}$ & 0.1768 & \multicolumn{4}{|c|}{0.3537} & 0.7074 & 1.4147 \\
\hline Frequency $f[\mathrm{~Hz}]$ & 1.0 & 1.0 & 5.0 & 10.0 & 20.0 & 1.0 & 1.0 \\
\hline $\begin{array}{c}\text { Dissipation factor } \\
\gamma[\%] \\
\end{array}$ & 30.86 & 7.64 & 7.82 & 8.22 & 3.37 & 7.73 & 3.89 \\
\hline $\begin{array}{c}\text { Damping ratio } \\
\zeta[-]\end{array}$ & 0.0246 & 0.0061 & 0.0062 & 0.0065 & 0.0027 & 0.0062 & 0.0031 \\
\hline
\end{tabular}

In the case of a model with viscous damping, the damping ratio $\zeta=c / c_{c r}$ is usually determined, where $c_{c r}$ is the critical damping. There is a relationship between the damping ratio $\zeta$ and the dissipation factor $\gamma[5]$ :

$$
\gamma=4 \pi \zeta \sqrt{1-\zeta^{2}}
$$

For a relatively small damping properties, the damping ratio is proportional to the dissipation factor, i.e. an approximate relationship is satisfied: $\zeta=\gamma / 4 \pi$. The estimated values of the damping ratio $\gamma$ for the tested material are shown in Table 2. In addition, for a sample with an area of $28.74 \mathrm{~cm}^{2}$, compressive forces induce appropriate stresses. It can be concluded that the damping parameters decrease with the increasing of compression.

\section{Analytical and numerical approach}

In the analytical approach, the reference structure was analyzed, i.e. a simple column fixed at the bottom. Natural frequencies have been derived assuming that the column made of IPE 140 profile with a height of $1.04 \mathrm{~m}$ is rigidly attached to the base, and a mass of 33.2 $\mathrm{kg}$ is at its top. The frequencies and modes of vibration were determined analytically, adopting the cantilever beam model, as well as numerically using two programs: ABAQUS/CAE and Robot ${ }^{\mathrm{TM}}$ Structural Analysis (Table 3).

Table 3. Natural frequencies for a rigidly supported column.

\begin{tabular}{|c|c|c|c|}
\hline Frequency & $\begin{array}{c}\text { Analytical } \\
\text { approach }\end{array}$ & Robot & ABAQUS \\
\hline$f_{y}[\mathrm{~Hz}]$ & 12.76 & 13.07 & 13.19 \\
\hline$f_{x}[\mathrm{~Hz}]$ & 44.27 & 45.36 & 43.90 \\
\hline
\end{tabular}

In the numerical approach, the spatial model was tested to determine modes of vibration (see Fig. 3) and to properly compare the results obtained with different methods. Figure 3 shows the modes obtained in the ABAQUS program. In addition to the modes along the $x$ and $y$ axes, there is another mode, torsional, related to the frequency whose value is between frequencies $f_{x}$ and $f_{y}$ (bending modes).

\section{Damping parameters}

In laboratory tests, the damping parameters of the structure were determined by examining the signal collected by the Sirius data acquisition system. For this purpose, the Dewesoft software was used, which automatically determines the modal parameters: natural 
frequencies and damping coefficients. In addition, the measured response signal was used to determine the logarithmic damping decrement.
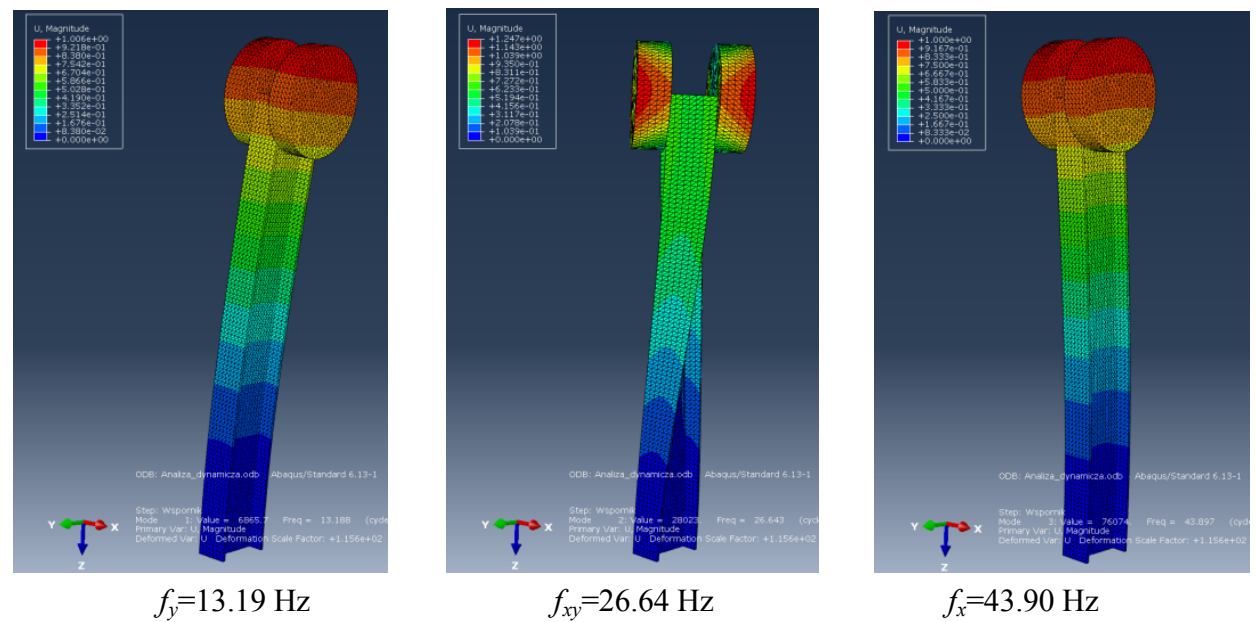

Fig. 3. The first three modes of vibration received in the ABAQUS program.

\subsection{Modal analysis}

In order to determine the modal parameters of the considered structure, the Sirius data acquisition system and the Dewesoft modal analysis software were used. Modal analysis is a way to study the dynamic properties of the considered systems in the frequency domain. Dynamic properties of the system are determined for specific frequencies, i.e. natural frequencies.

\subsubsection{Theoretical background}

The data acquisition system Dewesoft estimates the damping properties of analysed structure taking into account the transfer function and two different models for damping. For a structural damping model it is assumed, that the equation of motion for a single degree of freedom system (SDOF) is as follows:

$$
m \ddot{x}(t)+k x(t)+i h x(t)=F_{0} e^{i \omega t}
$$

where, $x(t)$ is the displacement, $F_{0}$ is the force amplitude, $m$ stands for mass, $k$ and $h$ are the elements of complex modulus, which could be interpreted as a storage and a loss modulus for considered material (i.e. $k^{*}=k+i h=k(1+i \eta)$ ), $i=\sqrt{-1}$ and $\omega$ is the natural frequency. In the experiment, the system response to the sinusoidal input given in the time domain is transferred to the frequency domain by Fourier transform. In this way, we get an output signal to a sinusoidal input of the same frequency but with different amplitude and phase. The frequency response function (FRF) is defined as the magnitude and phase difference between input and output sine waves at different frequencies. The so-called receptance of the frequency response function is defined as the ratio of the measured displacements $X(\omega)$ and force $F(\omega)$ :

$$
\alpha(\omega)=\frac{X(\omega)}{F(\omega)}=\frac{1}{k-\omega^{2} m+i h}=\frac{1}{\omega_{r}^{2}\left[1-\left(\frac{\omega}{\omega_{r}}\right)^{2}+i \eta_{r}\right]}
$$


where, $\eta_{\mathrm{r}}$ is the damping loss factor that corresponds to the resonance frequency $\omega_{\mathrm{r}}$. To plot the frequency response, we create a vector of frequencies $\mathbf{w}$ and compute the value of the transfer function $T F(i \mathbf{w})$ at those frequencies. Since the elements of transfer function are complex number, we can plot both its magnitude and phase as the Bode plots or we can plot its position in the complex plane as the Nyquist plot [6]. The receptance forms a perfect circle on the Nyquist plot, even if the multi-degrees of freedom systems are considered, because the contribution of other modes is irrelevant in the vicinity of the resonance. After fitting the circle to $N$ points around the resonance peak, one can take two points $\left(\omega_{b}, \omega_{a}\right)$ from this circle, below and above the resonant frequency $\omega_{r}$ and calculate the modal damping (loss factor $\eta_{r}$ ) as:

$$
\eta_{r}=\frac{\omega_{a}^{2}-\omega_{b}^{2}}{\omega_{r}^{2}\left[\tan \left(\theta_{a} / 2\right)-\tan \left(\theta_{b} / 2\right)\right]}=\frac{\omega_{2}^{2}-\omega_{1}^{2}}{2 \omega_{r}^{2}} \approx \frac{\omega_{2}-\omega_{1}}{\omega_{r}}
$$

where, $\omega_{1}$ and $\omega_{2}$ are the half power points of the considered FRF, i.e. $\alpha\left(\omega_{1}\right)=\alpha\left(\omega_{2}\right)=$ $\alpha\left(\omega_{r}\right) / \sqrt{2}$.

The equation of motion for SDOF system with a viscous damping model is

$$
m \ddot{x}(t)+c \dot{x}(t)+k x(t)=F_{0} \sin (\omega t)
$$

where, $\mathrm{c}(t)$ stands for the viscous damping parameter of considered system. In this case, the so-called mobility of the frequency response function is used, which is understood as the ratio of the measured velocity $\dot{X}(\omega)$ and force $F(\omega)$ :

$$
Y(\omega)=\frac{\dot{X}(\omega)}{F(\omega)}=\frac{i \omega}{k-\omega^{2} m+i \omega c}=\frac{i \omega}{\omega_{r}^{2}\left[1-\left(\frac{\omega}{\omega_{r}}\right)^{2}+2 i\left(\frac{\omega}{\omega_{r}}\right) \varsigma_{r}\right]}
$$

where, $\zeta_{r}=\mathrm{c} / \mathrm{c}_{c r}$ is the damping ratio which corresponds to the resonance frequency $\omega_{r}$, while $\mathrm{c}_{c r}$ is the critical damping. The mobility of FRF presented on the Nuquist plot leads to a convenient circle-fitting method that allowes determining the resonance frequency and damping ratio:

$$
\zeta_{r}=\frac{\omega_{a}^{2}-\omega_{b}^{2}}{2 \omega_{r}\left[\omega_{a} \tan \left(\theta_{a} / 2\right)+\omega_{b} \tan \left(\theta_{b} / 2\right)\right]}=\frac{\omega_{2}^{2}-\omega_{1}^{2}}{4 \omega_{r}^{2}} \approx \frac{\omega_{2}-\omega_{1}}{2 \omega_{r}}
$$

The symbols used in Eqn 7 are the same as in Eqn 4. In addition, there is a relationship between the damping loss factor and damping ratio: $\eta=2 \zeta$ [7].

\subsubsection{Results of the test}

The dynamic test was carried out on a steel structure, a column made of I-section (IPE 140) fixed at the bottom (see Fig. 4a) was selected. A mass of $33.2 \mathrm{~kg}$ was attached to the top of the column at the height of $1.04 \mathrm{~m}$. The connection to the base was made using M16 anchor bolts. During the test an additional polymer washers were placed under the column endplate (Fig. 4b). The dimensions of the endplate were 20x140x250 mm, and the polymer washers were $12 \mathrm{~mm}$ thick.

In the dynamic test, the relationship between the compressive stress value in the polymer and the dynamic parameters of the analyzed structure was investigated. The electronic torque wrench was used to control the torque as a bolt tension indicator. In this way, different values of the compressive stresses were obtained in polymer washers. 
a)

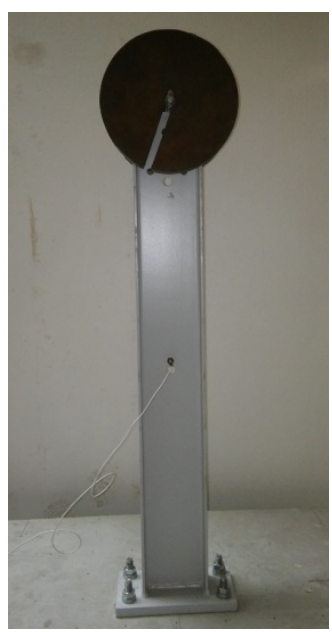

b)

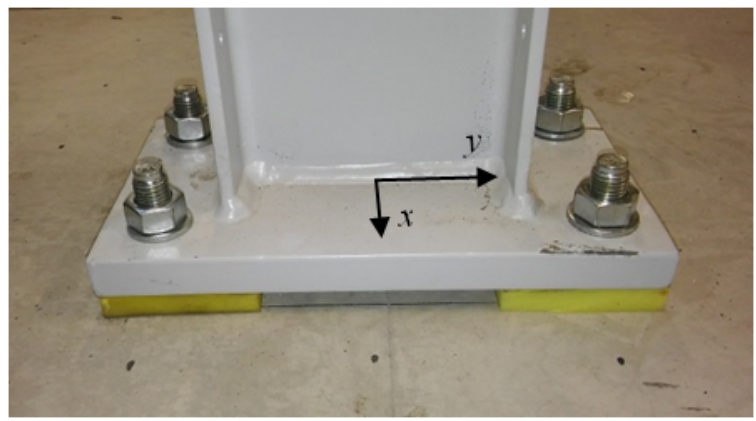

Fig. 4. Steel column with attached mass: a) view of the system, b) polymer washers in the node.

In order to obtain a similar compression stress in the polymer as in the previous test (Table 2), the required torque was calculated (Table 4).

Table 4. Relationship between torque, tension in the bolt and stress in the polymer.

\begin{tabular}{|c|c|c|c|c|c|}
\hline Torque, $\boldsymbol{M o}[\mathbf{N m}]$ & 4.92 & 9.85 & 19.69 & 39.39 & 78.77 \\
\hline Bolt tension, $\boldsymbol{F}[\mathrm{kN}]$ & 1.733 & 3.466 & 6.932 & 13.864 & 27.728 \\
\hline Compression, $\boldsymbol{\sigma}[\mathrm{MPa}]$ & 0.1768 & 0.3537 & 0.7074 & 1.4147 & 2.8294 \\
\hline
\end{tabular}

The torque value was calculated on the basis of the guidelines for M16 pretension bolts, class 8.8. The relationship between the preload and the torque was adopted for a coefficient of friction of 0.18 . In the dynamic test, the tension of the bolts was changed according to the data in Table 4, and thus an adequate compression in the polymer washers was obtained. The column was excited in two perpendicular planes with a modal hammer and the response of the structure in the form of acceleration was measured. Using the Dewesoft software and the modal identification function (MIF), the natural frequencies $\omega_{r}$ were determined. Then, using the receptance function (Eqn. 3) and the mobility function (Eqn. 6), the appropriate values of the loss factor $\eta_{r}$ and damping ratio $\zeta_{r}$ were determined (Table 5).

Table 5. Damping coefficients of the first mode for different compressive stresses in the polymer.

\begin{tabular}{|c|c|c|c|c|c|}
\hline $\begin{array}{c}\text { Compression } \\
\boldsymbol{\sigma}[\mathrm{MPa}]\end{array}$ & $\begin{array}{c}\text { Torque } \\
\boldsymbol{M o}[\mathbf{N m}]\end{array}$ & $\begin{array}{c}\text { Model of } \\
\text { damping }\end{array}$ & $\boldsymbol{f}_{\boldsymbol{y}}[\mathrm{Hz}]$ & $\boldsymbol{\eta}_{\boldsymbol{y}}[-]$ & $\boldsymbol{\zeta}_{\boldsymbol{y}}[-]$ \\
\hline 0.1768 & 4.29 & $\begin{array}{c}\text { structural } \\
\text { viscous }\end{array}$ & $\begin{array}{c}9.592 \\
9.257\end{array}$ & 0.2263 & 0.1150 \\
\hline 0.3537 & 9.85 & $\begin{array}{c}\text { structural } \\
\text { viscous }\end{array}$ & $\begin{array}{c}10.213 \\
10.314\end{array}$ & 0.1999 & 0.1020 \\
\hline 0.7074 & 19.69 & $\begin{array}{c}\text { structural } \\
\text { viscous }\end{array}$ & $\begin{array}{c}10.463 \\
10.460\end{array}$ & 0.1131 & 0.0625 \\
\hline 1.4147 & 39.39 & $\begin{array}{c}\text { structural } \\
\text { viscous }\end{array}$ & $\begin{array}{c}10.962 \\
10.815\end{array}$ & 0.0850 & 0.0425 \\
\hline
\end{tabular}


When a receptance function was used, a structural damping model was assumed, for the mobility function a viscous damping model was applied. It can be seen that the damping coefficients approximately meet the condition $\eta=2 \zeta$.

\subsection{Logarithmic decrement of damping}

The dynamic characteristics of the structure were also determined directly from the measured response signal, i.e. the acceleration function in the time domain. The use of a filter that narrows the signal to a resonant frequency leads to a sine wave function whose amplitude decreases over time. Taking the values of two successive amplitudes $q(t)$ and $q(t+T)$, one can calculate the logarithmic damping decrement:

$$
\delta_{r}=\ln \left(\frac{q(t)}{q\left(t+T_{r}\right)}\right)=\frac{2 \pi \zeta_{r}}{\sqrt{1-\zeta_{r}^{2}}}
$$

After calculating the damping decrement $\delta_{y}$, the value of the damping ratio $\zeta_{y}$ from the Eqn. 8 can be determined. The results for the first vibration mode and selected compressions in the base (Mo torque), with and without polymer, are shown in Figure 5.

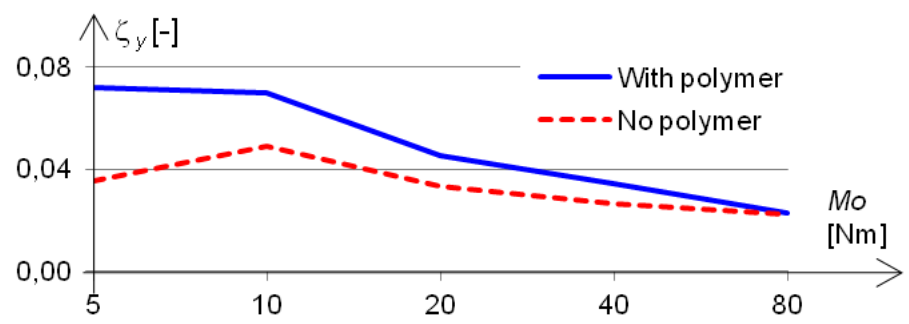

Fig. 5. The first mode damping ratio as a function of torque, causing force in the anchor bolt.

\section{Conclusions}

The paper presents several approaches and techniques for describing damping in structural systems. Damping coefficients: dissipation factor, loss factor, damping ratio and logarithmic decrement were estimated on the basis of data taken from laboratory measurements. The use of different calculation methods leads to slightly different results.

Moreover, the influence of polymer washers used in the butt nodes on the dynamic characteristics of considered structure was investigated. As expected, the increase in compressive stress in the polymer washers reduced their damping properties. But even with relatively high compression, the considered polymer has the ability to dissipate energy.

\section{References}

1. K. C. Chang, T. T. Soong, S. T. Oh, M. L. Lai, JSE, 121, 10, 1418-1425 (1995)

2. M. P. Singh, T. S. Chang, J. Eng. Mech., 135, 571-580 (2009)

3. G. P. Warn, K. L. Ryan, Buildings, 2, 300-325 (2012)

4. A. Banisheikholeslami, F. Behnamfar, M. Ghandil, JCSR 117, 185-195 (2016)

5. J. N. Libii, WIETE, 7, no1, 28-33 (2009)

6. W. S. Levine, The control handbook (CRC PRESS, IEEE PRESS, 1996)

7. FRF-modal test manual. www.dewesoft.com 